\title{
ON THE $p$-ADIC HEIGHTS OF SOME ABELIAN VARIETIES
}

\author{
HIDEO IMAI
}

\begin{abstract}
For an abelian variety defined over an algebraic number field, different definitions of $p$-adic heights have been given by several authors. In this note, we shall prove that the $p$-adic height defined by A. Néron and that by $\mathrm{P}$. Schneider coincide.
\end{abstract}

1. In this section we shall recall briefly the construction of $p$-adic heights. Let $K$ be a finite extension field of the $p$-adic rational number field $\mathbf{Q}_{p}$, where $p$ is a prime number. Let $\mathcal{O}$ be the integer ring of $K, \mathfrak{p}$ the maximal ideal of $\mathcal{O}$, and let $k=0 / \mathfrak{p}$ be the residue field of 0 . Let $A$ be an abelian variety of dimension $d$ defined over $K, \delta=\operatorname{id}_{A}$ the identity map of $A$. Let 0 be the identity point of $A$ and let $t_{1}, \ldots, t_{d}$ be a p-admissible system of local coordinates of $A$ at 0 in the sense of A. Néron; namely, $t_{1}, \ldots, t_{d}$ are $K$-rational functions on $A$ which constitute a local coordinate system at 0 and their reductions modulo $\mathfrak{p}$ are $k$-rational functions on $A \times \circ k$ also constitute a local coordinate system at the identity point of $A \times 0 k$.

Let $A(K)$ be the group of $K$-rational points of $A$ and let $U$ be an open neighborhood of 0 in $A(K)$. A function $\Phi: U \rightarrow K$ is called 0 -analytic on $U$ if for $a \in U$ we have

$$
\Phi(a)=\phi\left(t_{1}(a), \ldots, t_{d}(a)\right),
$$

where $\phi=\phi\left(\tau_{1}, \ldots, \tau_{d}\right)$ is a $d$-variable power series with coefficients in 0 which converges at $\left(t_{1}(a), \ldots, t_{d}(a)\right)$ for all $a \in U$.

A $K$-rational divisor $\Delta$ on $A$ is called disjoint from $0 \bmod \mathfrak{p}$ if any component of the set obtained from the reduction mod $\mathfrak{p}$ of the support of $\Delta$ does not contain the identity point of $A \times \circ k$.

For a subgroup $G$ of $A(K)$, let $\Lambda=\mathbf{Z}[G]$ be the group ring of $G$ with coefficients in $\mathbf{Z}$, which is also the group of 0 -cycles with components in $G$. Let $I \subset \Lambda$ be the augmentation ideal of $\Lambda$; i.e., the ideal generated by the cycles of the form (a) - (0) with $a \in G$. Let $I^{2} \subset \Lambda$ be the ideal generated by the cycles of the form $(a+b)-(a)-(b)+(0)$ with $a, b \in G$. As $(a+b)-(a)-(b)+(0)=$ $((a)-(0)) *((b)-(0))$, where $*$ is the multiplication in $\Lambda, I^{2}$ coincides with the square of $I ; I^{2}=\{\mathfrak{a} * \mathfrak{b} \mid \mathfrak{a}, \mathfrak{b} \in I\}$.

A divisor and a 0-cycle are called disjoint if their supports are disjoint. For a divisor $\Delta=\operatorname{div}(f)$ linearly equivalent to 0 , and a 0 -cycle $\mathfrak{a} \in I$ which are disjoint, we define a pairing $[\Delta, \mathfrak{a}]$ by $[\Delta, \mathfrak{a}]=\prod f\left(a_{i}\right)^{m_{i}}$ for $\mathfrak{a}=\sum m_{i}\left(a_{i}\right)$, as usual. For a divisor $\Delta$ algebraically equivalent to 0 and a 0 -cycle $\mathfrak{a}=\sum m_{i}\left(a_{i}\right)$, let $\Delta * a=\sum m_{i} \Delta_{a_{i}}$, where $\Delta_{a_{i}}$ is the translate of $\Delta$ by $a_{i}$, and let $\mathfrak{a}^{-}=\sum m_{i}\left(-a_{i}\right)$. If $\mathfrak{a} \in I$, then $\Delta * \mathfrak{a}$ is linearly equivalent to 0 . So for $\mathfrak{a}=\mathfrak{b} * \mathfrak{c} \in I^{2}, \Delta$ algebraically

Received by the editors August 7, 1985 and, in revised form, March 21, 1986.

1980 Mathematics Subject Classification (1985 Revision). Primary 14K15. 
equivalent to 0 , the value $\left[\Delta * \mathfrak{b}^{-}, \mathfrak{c}\right]$ has a meaning. We define the pairing $[\Delta, \mathfrak{a}]$ by $[\Delta, \mathfrak{a}]=\left[\Delta * \mathfrak{b}^{--}, \mathfrak{c}\right]$. By a certain reciprocity law, this pairing is known to be well defined.

In [4], A. Néron has shown the following fact: Let $G$ be an open subgroup of $A(K)$ such that $t_{i}(a) \in p \mathcal{O}$ for $a \in G, i=1, \ldots, d$, where $t_{1}, \ldots, t_{d}$ are $\mathfrak{p}$ admissible system of local coordinates of $A$ at 0 . Let $\Delta$ be a $K$-rational divisor on $A$ algebraically equivalent to 0 and disjoint from $0 \bmod \mathfrak{p}$. Then for $\mathfrak{a} \in I=I_{G}$,

$$
\theta_{\Delta, 0}^{\prime}(\mathfrak{a})=\lim _{\nu \rightarrow \infty}\left[\Delta, p^{\nu} \mathfrak{a}-p^{\nu} \delta \mathfrak{a}\right]^{1 / p^{\nu}}
$$

converges and satisfies $\theta_{\Delta, 0}^{\prime}(0)=1, \theta_{\Delta, 0}^{\prime}(\mathfrak{a})=[\Delta, \mathfrak{a}]$ for $\mathfrak{a} \in I^{2}$. The function $\theta_{\Delta, 0}^{\prime}(a)=\theta_{\Delta, 0}^{\prime}((a)-(0))$ on $a \in G$ is an 0 -analytic function with values in $1+p 0$ (cf. [4, Théorème and its proof]).

In general, for a $K$-rational divisior $\Delta$ not necessarily disjoint from $0 \bmod \mathfrak{p}$, take a $K$-rational function $f$ on $A$ such that $\operatorname{div}(f)+\Delta=\Delta^{\prime}$ is disjoint from $0 \bmod \mathfrak{p}$, and put $\theta_{\Delta, 0}^{\prime}(\mathfrak{a})=\theta_{\Delta^{\prime}, 0}^{\prime}(\mathfrak{a}) f(\mathfrak{a})^{-1}$.

REMARK. Note that for a divisor $\Delta=\operatorname{div}(f)$ linearly equivalent to 0 , Neron's $\mathfrak{p}$ adic theta function $\theta_{\Delta, 0}^{\prime}$ differs from the function $f$ slightly (cf. $[4, \S 4(\mathrm{~b})]$ ). So there are some ambiguities in the above definition of $\theta_{\Delta, 0}^{\prime}$ in the case of $\Delta$ not disjoint from $0 \bmod \mathfrak{p}$. However from op. cit., for $\mathfrak{a} \in I, \theta_{\Delta, 0}^{\prime}(\mathfrak{a})$ is defined up to units so this ambiguity disappears when we take the image of $\theta_{\Delta, 0}^{\prime}(\mathfrak{a})$ by a homomorphism which is trivial on the unit group.

In $\S 3$ we shall prove the following Proposition.

PROPOSITION. Let $\Omega$ be the completion of the algebraic closure of $K, R$ the integer ring of $\Omega$, and $\mathfrak{m}$ the maximal ideal of $R$. Let $\hat{A}(R)=\left\{a \in A(\Omega) \mid t_{i}(a) \in \mathfrak{m}\right.$, $i=1, \ldots, d\}$. Assume that $A$ has ordinary good reduction mod $\mathfrak{p}$. Let $\Delta$ be a $K$ rational divisor on $A$ algebraically equivalent to 0 and disjoint from $0 \bmod \mathfrak{p}$. Then the limit

$$
\theta_{\Delta, 0}^{\prime}(\mathfrak{a})=\lim _{\nu \rightarrow \infty}\left[\Delta, p^{\nu} \mathfrak{a}-p^{\nu} \delta \mathfrak{a}\right]^{1 / p^{\nu}}
$$

converges for $\mathfrak{a}=(a)-(0)$ with $a \in \hat{A}(R)$, and $\theta_{\Delta, 0}^{\prime}(a)=\theta_{\Delta, 0}^{\prime}((a)-(0))$ defines an O-analytic function on $\hat{A}(R)$ with values in $1+\mathfrak{m}$, satisfying $\theta_{\Delta, 0}^{\prime}(\mathfrak{a})=[\Delta, \mathfrak{a}]$ for $\mathfrak{a} \in I^{2}=I_{\hat{A}(R)}^{2}$. Moreover, $\theta_{\Delta, 0}^{\prime}$ is the unique 0 -analytic function on $\hat{A}(R)$ which satisfies $\theta_{\Delta, 0}^{\prime}(\mathfrak{a})=[\Delta, \mathfrak{a}]$ for $\mathfrak{a} \in I^{2}$.

A. Néron has extended his $\mathfrak{p}$-adic theta function $\theta_{\Delta, 0}^{\prime}$ to $A(K)$ in the following way. Let $\Delta$ be a $K$-rational divisor on $A$ algebraically equivalent to 0 and let $\mathfrak{a}=(a)-(0)$ be a 0 -cycle disjoint from $\Delta$ with $a \in A(K)$. Let $m$ be the exponent of the finite group $A(K) / G$ ( $G$ is an open subgroup of $A(K)$ such that $t_{i}(a) \in p 0$ for $a \in G, i=1, \ldots, d)$. Then define the extension $\tilde{\theta}_{\Delta, 0}^{\prime}$ of $\theta_{\Delta, 0}^{\prime}$ by

$$
[\Delta, m \mathfrak{a}-m \delta \mathfrak{a}]=\tilde{\theta}_{\Delta, 0}^{\prime}(\mathfrak{a})^{m} \theta_{\Delta, 0}^{\prime}(m \delta \mathfrak{a})^{-1} .
$$

So $\tilde{\theta}_{\Delta, 0}^{\prime}(a)=\tilde{\theta}_{\Delta, 0}^{\prime}((a)-(0))$ is defined up to $m$ th root of unity (with value in the algebraic closure of $K)$.

Now let $K$ be an algebraic number field of finite degree. Let $A$ be an abelian variety defined over $K$. For a nonarchimedean prime $v$ of $K$, let $K_{v}$ be the completion of $K$ at $v, O_{v}$ the integer ring of $K_{v}, \mathfrak{p}_{v}$ the maximal ideal of $\mathcal{O}_{v}$, and let 
$k_{v}=\mathcal{O}_{v} / \mathfrak{p}_{v}$ be the residue field at $v$. Let $\mathbf{A}_{K}^{\times}$be the group of finite ideles of $K$; i.e., $\mathbf{A}_{K}^{\times}$is the restricted direct product $\Pi^{\prime} K_{v}^{\times}$where the product ranges through all of the nonarchimedean primes of $K$ and $\left(x_{v}\right)$ is in $\mathbf{A}_{K}^{\times}$if $x_{v} \in \mathcal{O}_{v}^{\times}$for all but a finite number of primes $v$. We have a canonical homomorphism $K^{\times} \rightarrow \mathbf{A}_{K}^{\times}$and we identify $K^{\times}$with its image. Let $p$ be a fixed prime number such that $A$ has ordinary good reduction at all primes of $K$ over $p$. Let $\rho: \mathbf{A}_{K}^{\times} \rightarrow \mathbf{Z}_{p}$ be a nontrivial continuous homomorphism which is trivial on $K^{\times}$corresponding to a $\mathbf{Z}_{p}$-extension of $K$ by the class field theory. For a nonarchimedean prime $v$, let $\rho_{v}$ be the component of $\rho$ at $v$.

Let $\Delta$ be a $K$-rational divisor on $A$ algebraically equivalent to 0 and $a \in A(K)$, which are disjoint. For a nonarchimedean prime $v$, we can construct the $v$-adic theta function $\tilde{\theta}_{\Delta, 0, v}^{\prime}(a)$ substituting $A, K_{v}$ instead of $A, K$ in the above construction.

Then A. Néron's $p$-adic height corresponding to the homomorphism $\rho$ is defined by

$$
h_{\Delta, \rho}(a)=\sum \rho_{v}\left(\tilde{\theta}_{\Delta, 0, v}^{\prime}(a)\right)
$$

where the summation ranges through all of the nonarchimedean primes of $K$, and as $\tilde{\theta}_{\Delta, 0, v}^{\prime}(a)^{m} \in K_{v}^{\times}$for some $m$, we put

$$
\rho_{v}\left(\tilde{\theta}_{\Delta, 0, v}^{\prime}(a)\right)=\frac{1}{m} \rho_{v}\left(\tilde{\theta}_{\Delta, 0, v}^{\prime}(a)^{m}\right) .
$$

It can be shown that this function is well defined and depends only on the linear equivalence class of $\Delta$.

Let $A^{\prime}=\operatorname{Pic}^{0}(A)$ be the dual abelian variety. Then we obtain a pairing from $A(K) \times A^{\prime}(K)$ to $\mathbf{Q}_{p}$ by putting

$$
\langle a, \operatorname{cl}(\Delta)\rangle_{N}=h_{\Delta, \rho}(a),
$$

here $\operatorname{cl}(\Delta)$ is the image of $\Delta$ in $A^{\prime}$.

Next we recall the construction of P. Schneider's $p$-adic height pairing (as P. Schneider's $p$-adic height pairing coincides with Mazur and Tate's $p$-adic height pairing (cf. $[3, \S 4.4]$ ), we recall the latter).

Let $K$ be an algebraic number field of finite degree and $A$ an abelian variety defined over $K$. Let $O$ be the integer ring of $K$ and let $A$ be the Néron model of $A$ over 0 . Let $A^{0}$ be the connected component of the identity of $A$ and let $A^{\prime}$ be the dual abelian variety of $A$. For a $K$-rational divisor $\Delta$ of $A$ algebraically equivalent to 0 there corresponds an extension $X$ of $\AA^{0}$ by the multiplicative group $\mathbf{G}_{m}$ over O (cf. $[1, \S 1]$ );

$$
0 \rightarrow \mathbf{G}_{m} \rightarrow X \stackrel{\pi}{\rightarrow} \AA^{0} \rightarrow 0 .
$$

Here we note that for an extension field $L$ of $K$, the group of $L$-rational points $X(L)$ of $X$ may be described as follows (cf. [3, p. 210]): $X(L)$ is the set of pairs $(\mathfrak{a}, c)$ where $\mathfrak{a}$ is a 0 -cycle of degree 0 with $L$-rational components and $c \in L^{\times}$; $c(\mathfrak{a}, 1)=(\mathfrak{a}, c),\left(\mathfrak{a}_{1}, c_{1}\right)\left(\mathfrak{a}_{2}, c_{2}\right)=\left(\mathfrak{a}_{1}+\mathfrak{a}_{2}, c_{1} c_{2}\right)$, when $(\mathfrak{a}, c)$ maps to $0 \in A(L)$ (i.e., when $\mathfrak{a} \in I^{2}$ is Néron's notation recalled as before), then $(\mathfrak{a}, c)=(0,[\Delta, \mathfrak{a}] c)$. For $a \in A(L)$, the map $\sigma_{\Delta}: a \mapsto((a)-(0), 1)$ defines a $K$-rational section of $\pi$, and $\sigma_{\Delta}$ is regular on $A(L) \backslash|\Delta|$.

Let $p$ be a fixed prime number such that $A$ has ordinary good reduction at the primes of $K$ over $p$. Let $\rho: \mathbf{A}_{K}^{\times} \rightarrow \mathbf{Z}_{p}$ be a nontrivial continuous homomorphism 
which is trivial on $K^{\times}$corresponding to a $\mathbf{Z}_{p}$-extension of $K$, where $\mathbf{A}_{K}^{\times}$is the group of finite ideles of $K$ as before. For a nonarchimedean prime $v$ of $K$, let $\rho_{v}$ be the component of $\rho$ at $v$.

By considering the $K_{v}$-rational points of (1) we obtain the following exact sequence:

$$
0 \rightarrow K_{v}^{\times} \rightarrow X\left(K_{v}\right) \rightarrow A\left(K_{v}\right) \rightarrow 0
$$

(note that $H^{1}\left(R, \mathbf{G}_{m}\right)=0$ for a local ring $R$ ). In [3, Theorem (1.5)], Mazur and Tate have constructed a canonical $\rho_{v}$-splitting $\psi_{\rho, v}: X\left(K_{v}\right) \rightarrow \mathbf{Q}_{p}$ for the above exact sequence (in our case, canonical $\rho_{v}$-splitting is the restriction to the fiber at $\operatorname{cl}(\Delta) \in A^{\prime}(K)$ of their canonical $\rho_{v}$-splitting of the biextension of $A\left(K_{v}\right) \times A^{\prime}\left(K_{v}\right)$ by $\left.K_{v}^{\times}\right)$. We recall that a homomorphism $\psi_{\rho, v}: X\left(K_{v}\right) \rightarrow \mathbf{Q}_{p}$ is called a $\rho_{v}$-splitting if it satisfies

$$
\psi_{\rho, v}(c x)=\rho_{v}(c)+\psi_{\rho, v}(x) \quad \text { for } c \in K_{v}^{\times}, x \in X\left(K_{v}\right) .
$$

(The characterizing property of canonicalness will be explained later.)

Now let $a \in A(K)$ and take a point $x \in X(K)$ mapping to $a$. Mazur and Tate have defined their $p$-adic height pairing by

$$
\langle a, \operatorname{cl}(\Delta)\rangle_{S}=\sum \psi_{\rho, v}(x),
$$

where the summation ranges through the nonarchimedean primes of $K$. Here we note that the sum on the right is well defined and independent of the choice of $x$ and depends only on the linear equivalence class of $\Delta$.

2. The result of this note is the following Theorem.

THEOREM. Let $K$ be an algebraic number field of finite degree and $A$ an abelian variety defined over $K$. Let $p$ be a prime number such that $A$ has ordinary good reduction at all primes of $K$ over $p$. Let $\rho: \mathbf{A}_{K}^{\times} \rightarrow \mathbf{Z}_{p}$ be a nontrivial continuous homomorphism trivial on $K^{\times}$, corresponding to a $\mathbf{Z}_{p}$-extension of $K$. Then for $a \in A(K)$ and $\Delta a K$-rational divisior on $A$ algebraically equivalent to 0 , we have

$$
\langle a, \operatorname{cl}(\Delta)\rangle_{N}=\langle a, \operatorname{cl}(\Delta)\rangle_{S} .
$$

PROOF. Let the notations be the same as in $\S 1$. Let $v$ be a nonarchimedean prime of $K$. We take a divisor from the linear equivalence class of $\Delta$, which is disjoint from $0 \bmod v$ for all primes $v$ over $p$ (for simplicity, we write this divisor by $\Delta$ ). We compare the local components of Néron's and Schneider's $p$-adic height pairings.

First we treat the case where $v$ is a prime not above $p$. In this case $\rho_{v}$ is trivial on $O_{v}^{\times}$as $\rho$ is continuous. Let $q$ be the residue characteristic of $v$, and let $G$ be an open subgroup of $A^{0}\left(O_{v}\right)$ such that $t_{i}(a) \in q \mathcal{O}_{v}$ for $a \in G, i=1, \ldots, d$; here $t_{1 \text { it }, 6, ;} t_{d}$ are $v$-admissible systems of local coordinates of $A$ at 0 .

If $\Delta=\operatorname{div}(f)$ is linearly equivalent to 0 , then from $[3,(2.2 .2)]$, we have $\psi_{\rho, v}((\mathfrak{a}, 1))=\rho_{\boldsymbol{v}}(f(\mathfrak{a}))$ for $\mathfrak{a} \in I=I_{G}$, whenever the right-hand side is defined. On the other hand from the Remark before the Proposition in $\S 1$ (cf. also [4, $\S 4(\mathrm{~b})]$ ), $\rho_{v}\left(\theta_{\Delta, \boldsymbol{d}}^{\prime}(\mathfrak{a})\right)=\boldsymbol{p}_{v}(f(\mathfrak{a}))$

In general let $\Delta$ be a divisor algebraically equivalent to 0 . Take a $K_{v}$-rational function on A such that $\operatorname{div}(f)+\Delta=\Delta^{\prime}$ is disjoint from 0 mod $v$. We consider the objects of 1 defined with respect to $\Delta^{\prime}$. Now in the case we are considering, the 
characterizing property of canonicalness of $\rho_{v}$-splitting is that $\psi_{\rho, v}=0$ on $X\left(O_{v}\right)$, where $X$ is the extension of $A^{0}$ by $\mathbf{G}_{m}$ corresponding to $\Delta^{\prime}$. Let $m=\left[A\left(K_{v}\right): G\right]$, where $G$ is an open subgroup of $A^{0}\left(O_{v}\right)$ as above. Then we have for $\mathfrak{a}=(a)-(0)$, $a \in A\left(K_{v}\right)$.

$$
\begin{aligned}
\psi_{\rho, v}((\mathfrak{a}, 1)) & =\frac{1}{m} \psi_{\rho, v}((m \mathfrak{a}, 1)) \\
& =\frac{1}{m} \psi_{\rho, v}\left(\left(0,\left[\Delta^{\prime}, m \mathfrak{a}-m \delta \mathfrak{a}\right]\right)\right)+\frac{1}{m} \psi_{\rho, v}((m \delta \mathfrak{a}, 1)) \\
& =\frac{1}{m} \rho_{v}\left(\left[\Delta^{\prime}, m \mathfrak{a}-m \delta \mathfrak{a}\right]\right),
\end{aligned}
$$

as $m \delta \mathfrak{a}$ has support in $A^{0}\left(O_{v}\right)$.

On the other hand from the equation defining the $v$-adic theta function $\tilde{\theta}_{\Delta^{\prime}, 0, v}^{\prime}$, we have

$$
\frac{1}{m} \rho_{v}\left(\left[\Delta^{\prime}, m \mathfrak{a}-m \delta \mathfrak{a}\right]\right)=\rho_{v}\left(\tilde{\theta}_{\Delta^{\prime}, 0, v}^{\prime}(\mathfrak{a})\right),
$$

as $\theta_{\Delta^{\prime}, 0, v}^{\prime}(m \delta \mathfrak{a}) \in 1+q \bigcirc_{v}$.

From the multiplicativity of the local pairings with respect to $\Delta$, we see that in this case the local components of Néron's and Schneider's $p$-adic height pairings coincide.

In the case when $v$ is a prime over $p$, we consider the fiber at $v$ of the exact sequence (1). Taking the formal completion along the maximal torus of the special fiber (we denote this completion by superscript $t$ ) we obtain the following exact sequence (cf. $[3, \S 5])$

$$
0 \rightarrow \mathrm{G}_{m}^{t} \rightarrow X^{t} \rightarrow A^{t} \rightarrow 0 .
$$

Mazur and Tate have proved that the above exact sequence has a unique splitting $\psi: X^{t} \rightarrow \mathbf{G}_{m}^{t}$. The characterizing property of canonicalness of $\rho_{v}$-splitting is that $\psi_{\rho, v}=\rho_{v} \circ \psi$ on $X^{t}\left(O_{v}\right)$. Now take the formal completion along the 0 -section of the special fiber of the exact sequence (1) (we denote this completion by $\hat{X}$ etc.); we obtain the following exact sequence:

$$
0 \rightarrow \hat{\mathbf{G}}_{m} \rightarrow \hat{X} \rightarrow \hat{A} \rightarrow 0 .
$$

Let $\Omega_{v}$ be the completion of the algebraic closure of $K_{v}, R_{v}$ the integer ring of $\Omega_{v}$, and let $\mathfrak{m}_{v}$ be the maximal ideal of $R_{v}$. Considering the $R_{v}$-valued points we obtain the following exact sequence:

$$
0 \rightarrow 1+\mathfrak{m}_{v} \rightarrow \hat{X}\left(R_{v}\right) \rightarrow \hat{A}\left(R_{v}\right) \rightarrow 0 .
$$

From Mazur and Tate's splitting $\psi: X^{t} \rightarrow \mathbf{G}_{m}^{t}$, we obtain a splitting $\psi^{\prime}: \hat{X}\left(R_{v}\right) \rightarrow$ $1+\mathfrak{m}_{v}$ of the above exact sequence. Now $\Delta$ is disjoint from $0 \bmod v$. From the Proposition in $\S 1$, for $\mathfrak{a}=(a)-(0)$ with $a \in \hat{A}\left(R_{v}\right)$,

$$
\theta_{\Delta, 0, v}^{\prime}(\mathfrak{a})=\lim _{\nu \rightarrow \infty}\left[\Delta, p^{\nu} \mathfrak{a}-p^{\nu} \delta \mathfrak{a}\right]^{1 / p^{\nu}}
$$

converges and defines an $\mathcal{O}_{v}$-analytic function on $\hat{A}\left(R_{v}\right)$. From $\theta_{\Delta, 0, v}^{\prime}(\mathfrak{a})=[\Delta, \mathfrak{a}]$ for $\mathfrak{a} \in I^{2}$, by putting $\mathfrak{a}=(a+b)-(a)-(b)+(0)$ with $a, b \in \hat{A}\left(R_{v}\right)$ we have

$$
\theta_{\Delta, 0, v}^{\prime}(a+b) \theta_{\Delta, 0, v}^{\prime}(a)^{-1} \theta_{\Delta, 0, v}^{\prime}(b)^{-1}=[\Delta, \mathfrak{a}]=\sigma_{\Delta}(a+b) \sigma_{\Delta}(a)^{-1} \sigma_{\Delta}(b)^{-1} ;
$$


here $\sigma_{\Delta}: a \mapsto((a)-(0), 1)$ is the $K$-rational section of $\pi: X \rightarrow A$ such that $\sigma_{\Delta}(0)=1$. Hence $\sigma_{\Delta} \cdot \theta_{\Delta, 0, v}^{\prime-1}: \hat{A}\left(R_{v}\right) \rightarrow \hat{X}\left(R_{v}\right)$ is a section of (2) as abstract groups. As $\theta_{\Delta, 0, v}^{\prime}$ is $O_{v}$-analytic and $\sigma_{\Delta}$ is $K$-rational, from [8, (4.2), Corollary 1], $\sigma_{\Delta} \cdot \theta_{\Delta, 0, v}^{\prime-1}$ is in fact a formal group homomorphism defined over $\mathcal{O}_{v}$. We define $\psi^{\prime \prime}: \hat{X}\left(R_{v}\right) \rightarrow 1+\mathfrak{m}_{v}$ by

$$
\psi^{\prime \prime}(x)=x /\left(\sigma_{\Delta} \cdot \theta_{\Delta, 0, v}^{\prime-1}(\pi(x))\right),
$$

then $\psi^{\prime \prime}$ is a splitting of (2) as abstract groups. As $\psi^{\prime} \cdot \psi^{\prime \prime}-1$ is trivial on $1+\mathfrak{m}_{v}$, it induces a formal group homomorphism $\psi^{\prime} \cdot \psi^{\prime \prime}-1: \hat{A} \rightarrow \hat{\mathbf{G}}_{m}$ defined over $O_{v}$ (cf. ibid.). We need the following Lemma.

LEMMA. Let $\hat{A}$ be the formal group of an abelian variety defined over $K_{v}$, which has good reduction mod $v$. Then any formal group homomorphism $\phi: \hat{A} \rightarrow \hat{\mathbf{G}}_{m}$ defined over $\mathrm{O}_{v}$ is trivial.

PROOF OF THE LEMMA. If $\phi$ were nontrivial, there should exist a surjective homomorphism $\psi$ such that $\phi=\psi^{m}$ for some $m$ (cf. [8, (4.2), Corollary 1]). The reduction mod $v$ of the homomorphism $\psi$ is a surjective homomorphism of formal groups over $k_{v}, \psi / k_{v}: \hat{A} / k_{v} \rightarrow \hat{\mathbf{G}}_{m} / k_{v}$. Consider the characteristic polynomials of the Frobenius endomorphism of $\bar{k}_{v}$ over $k_{v}$ acting on these $p$-divisible groups over $k_{v}$ (cf. $[2, \S 4 . e]$ ). The multiplicativity of the characteristic polynomials and the Riemann-Weil hypothesis lead to a contradiction.

By the Lemma, $\psi^{\prime}=\psi^{\prime \prime}$. We apply the Mazur-Tate's construction of $p$-adic height pairing recalled as before. Let $m=\left[A\left(K_{v}\right): \hat{A}\left(O_{v}\right)\right]$, which is the cardinality of the group of rational points of the special fiber of $A$ at $v$. As before, let $\mathfrak{a}=$ $(a)-(0)$. Then we have

$$
\begin{aligned}
\psi_{\rho, v}((\mathfrak{a}, 1)) & =\frac{1}{m} \psi_{\rho, v}((m \mathfrak{a}, 1)) \\
& =\frac{1}{m} \rho_{v}([\Delta, m \mathfrak{a}-m \delta \mathfrak{a}])+\frac{1}{m} \rho_{v} \circ \psi^{\prime}((m \delta \mathfrak{a}, 1)) \\
& =\frac{1}{m} \rho_{v}([\Delta, m \mathfrak{a}-m \delta \mathfrak{a}])+\frac{1}{m} \rho_{v} \circ \psi^{\prime \prime}\left(\sigma_{\Delta}(m a)\right) \\
& =\frac{1}{m} \rho_{v}([\Delta, m \mathfrak{a}-m \delta \mathfrak{a}])+\frac{1}{m} \rho_{v} \circ \theta_{\Delta, 0, v}^{\prime}(m a) \\
& =\rho_{v}\left(\tilde{\theta}_{\Delta, 0, v}^{\prime}(\mathfrak{a})\right) .
\end{aligned}
$$

So $\psi_{\rho, v}((\mathfrak{a}, 1))=\rho_{v}\left(\tilde{\theta}_{\Delta, 0, v}^{\prime}(\mathfrak{a})\right)$ for all nonarchimedean primes $v$. The Theorem is proved.

3. In this section we prove the Proposition of $\S 1$. Let the notations be the same as in the Proposition. Let $f$ be the $K$-rational function on $A$ such that $\operatorname{div}(f)=(p \delta)^{-1} \Delta-p \Delta, f(0)=1$. We expand $f$ as a power series in $t_{1}, \ldots, t_{d}$ near 0 , then from $[4$, p. 158$]$, we see that $f \in \mathcal{O}\left[\left[t_{1}, \ldots, t_{d}\right]\right]$ and $f-1 \equiv 0(\bmod \operatorname{deg} 2)$. Also from the arguments used in ibid. p. 158, to prove the convergence of $\theta_{\Delta, 0}^{\prime}$, it suffices to show that for $a \in \hat{A}(R) \operatorname{ord}_{p}\left(\left(f\left(p^{\nu} a\right)-1\right) / p^{\nu}\right)$ diverges to $\infty$ as $\nu \rightarrow \infty$, where $\operatorname{ord}_{p}$ is the additive valuation such $\operatorname{ord}_{p}(p)=1$. Let $\hat{A}$ be the formal group of $A$, and let $I$ be the integer ring of the completion of the maximal unramified 
extension of $K$. Then $\hat{A}$ is isomorphic to $\hat{\mathbf{G}}_{m}^{d}$ over $I$. Hence $\hat{A}(R) \simeq(1+\mathfrak{m})^{d}$ over $I$. As this isomorphism may be expressed by some invertible power series, we see that $\operatorname{ord}_{p}\left(t_{i}\left(p^{\nu} a\right)\right)=\operatorname{ord}_{p}\left((1+z)^{p^{\nu}}-1\right)$ for some $z \in \mathfrak{m}$. We expand $(1+z)^{p^{\nu}}$ with binomial coefficients $\left(\begin{array}{c}p^{\nu} \\ r\end{array}\right)$. For $r<p^{\nu-1}$, using the fact that $\operatorname{ord}_{p}(r !)=\sum_{k=0}^{\infty}\left[r / p^{k}\right]$, where $[x]$ is the largest integer such that $[x] \leq x$, after some computations we have $\operatorname{ord}_{p}\left(\begin{array}{c}p^{\nu} \\ r\end{array}\right)=\nu-\operatorname{ord}_{p}(r)$. Hence

$$
\operatorname{ord} p\left((1+z)^{p^{\nu}}-1\right) \geq \operatorname{Min}\left(p^{\nu-1} \operatorname{ord}_{p} z, \nu-\operatorname{ord}_{p} r+r \operatorname{ord}_{p} z\right),
$$

where $r$ ranges from 1 to $p^{\nu-1}-1$.

As $\operatorname{ord}_{p}\left(t_{i}\left(p^{\nu} a\right)\right)$ satisfies similar inequality, we see that

$$
\operatorname{ord}_{p}\left(\left(f\left(p^{\nu} a\right)-1\right) / p^{\nu}\right) \geq \operatorname{Min}\left(2 p^{\nu-1} \operatorname{ord}_{p}\left(z_{i}\right)-\nu, \nu-2 \operatorname{ord}_{p} r+2 r \operatorname{ord}_{p}\left(z_{i}\right)\right),
$$

where $r$ ranges from 1 to $p^{\nu-1}-1, i$ ranges from 1 to $d$, and $z_{i}$ are elements in $\mathrm{m}$.

From $\operatorname{ord}_{p}\left(z_{i}\right)>0$, we see easily that the right-hand side of the above inequality diverges to $\infty$ when $\nu \rightarrow \infty$.

When $\operatorname{ord}_{p}\left(z_{i}\right)$ ranges through a compact subset of $\{x \in \mathbf{R} \mid x>0\}$, this conver-

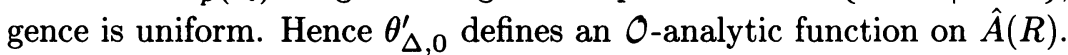

Now let $\mathfrak{a}=(a+b)-(a)-(b)+(0) \in I^{2}$. By Néron's result we have $\theta_{\Delta, 0}^{\prime}(\mathfrak{a})=$ $[\Delta, \mathfrak{a}]$ for $a, b \in \hat{A}(R)$ sufficiently close to 0 (cf. [4, Théorème]). For a fixed $b$, both sides of the above equation are given by power series in coordinates of $a$. Hence we have $\theta_{\Delta, 0}^{\prime}(\mathfrak{a})=[\Delta, \mathfrak{a}]$ for all $\mathfrak{a} \in I^{2}$. Uniqueness is clear from the Lemma in $\S 2$.

\section{REFERENCES}

1. S. Bloch, A note on height pairings, Tamagawa numbers and the Birch and Suinnerton-Dyer conjecture, Invent. Math. 58 (1980), 65-76.

2. B. Mazur, Rational points of abelian varieties with values in towers of number fields, Invent. Math. 18 (1972), 183-266.

3. B. Mazur and J. Tate, Canonical height pairing via biextensions, Arithmetic and Geometry (Volume dedicated to Shafarevich, vol. 1), Progress in Math., vol. 35, Birkhäuser, Boston, Basel, and Stuttgart, 1983, pp. 195-273.

4. A. Néron, Fonctions thêta p-adiques et hauteurs p-adiques (Séminaire de théorie de nombres, Paris 1980-1981), Progress in Math., vol. 22, Birkhäuser, Boston, Basel, and Stuttgart, 1982, pp. $149-174$.

5. J. Oesterlé, Construction de hauteurs archimediennes et p-adiques suivant la methode de Bloch (Séminaire de théorie de nombres, Paris 1980-1981), Progress in Math., vol. 22, Birkhäuser, Boston, Basel, and Stuttgart, 1982, pp. 175-192.

6. B. Perrin-Riou, Hauteurs p-adiques (Séminaire de théorie de nombres, Paris 1982-1983), Progress in Math., vol. 51, Birkhäuser, Boston, Basel, and Stuttgart, 1984, pp. 233-257.

7. P. Schneider, p-adic height pairings. I, Invent. Math. 69 (1982), 401-409.

8. J. Tate, p-divisible groups, Proceedings of a Conference on Local Fields, NUFFIC Summer School held at Driebergen, 1966, Springer, Berlin, Heidelberg, and New York, 1967, pp. 158-183.

Department of Mathematics, College of General Education, Tôhoku UniVERSITY, SENDAI, 980, JAPAN 